\title{
Dilute Solution Properties of Water-Soluble Incompletely Substituted Cellulose Acetate
}

\author{
Kenji Kamide, Masatoshi SaIto, and Tatsuyuki ABE \\ Textile Research Laboratory, Asahi Chemical Industry Co., Ltd., \\ Hachonawate, Takatsuki, Osaka 569, Japan.
}

(Received June 23, 1980)

\begin{abstract}
Water-soluble incompletely substituted cellulose acetate (CA) was synthesized by the hydrolysis of cellulose diacetate with hydrochloric acid. CA having a degree of substitution $D S$ between 0.39 and 0.81 was water-soluble. The CA $(D S=0.49)$ whole polymer was fractionated into 15 parts, using water-methanol as the solvent-nonsolvent by the successive solution method. The $\mathrm{CA}(D S=0.49)$ polymer was soluble in highly polar organic solvents. Solution viscosity, light scattering, and membrane osmometry measurements were performed at $25 \mathrm{C}$ on these fractions in $N, N$-dimethylacetamide (DMAc), dimethyl sulfoxide (DMSO), water, and formamide. The fractions had ratios of weight-to-number-average molecular weight $M_{w} / M_{n}$ of approximately 1.3 , independent of $M_{w}$. The following relations between limiting viscosity number [ $\left.\eta\right], z$-average radius of gyration $\left\langle S^{2}\right\rangle_{z}^{1 / 2}$ and $M_{w}$ were obtained: $[\eta]=0.19 M_{w}^{0.60}\left(\mathrm{~cm}^{3} \mathrm{~g}^{-1}\right)$ and $\left\langle S^{2}\right\rangle_{z}^{1 / 2}=0.50 \times 10^{-8}$ $M_{w^{\prime}}^{0.52}(\mathrm{~cm})$ in DMAc at $25^{\circ} \mathrm{C}$. Flory's viscosity parameter $\Phi$ in DMAc was $(1.82-2.18) \times 10^{23}$, showing a small molecular weight dependence. The short-range interaction parameter $\mathrm{A}$ was estimated thermodynamically as $1.33 \times 10^{-8}(\mathrm{~cm})$ in DMAc and the conformation parameter $\sigma$ found to be 2.29. The effect of DS on the molecular characteristics of cellulose acetate is discussed.

KEY WORDS Cellulose Acetate / Solution Property / Unperturbed Chain

Dimension / Mark-Houwink-Sakurada Equation / Conformation

Parameter /
\end{abstract}

For years the dilute solution properties of cellulose and its derivatives have been an important target of attention in polymer science. In spite of this, the effect of the degree of substitution $(D S)$ on these properties has been studied only in part for cellulose nitrate and cellulose acetate, particularly in the limited range of higher $D S$. For example, Moore and his co-workers ${ }^{1}$ established parameters in the Mark-Houwink-Sakurada (MHS) equation [eq 1] for cellulose nitrate with nitrogen content $\mathrm{N} \%=12.4-13.8$ in various solvents. Schulz and Penzel $^{2-4}$ measured the solution viscosity and light scattering of cellulose nitrates $(\mathrm{N}=12.9$ and $13.9 \%)$ in acetone and ethyl acetate and estimated the thermodynamic and hydrodynamic parameters. Recently, we determined the molecular parameters of cellulose acetate ( $D S=2.46$ and 2.92) on the basis of solution viscosity, gel-permeation chromatography, sedimentation velocity, membrane osmometry, and light scattering. ${ }^{5-8}$ We found the unper- turbed chain dimension to be larger in cellulose acetate $(D S=2.46)$ than in cellulose acetate $(D S=2.92)$ and that $\mathrm{A}$ of cellulose acetate ( $D S=2.46)$ is markedly dependent on the solvent nature (i.e., dielectric constant). These features were interpreted in terms of the polar character of cellulose acetate $(D S=2.46)$. Unfortunately, no work has been carried out on cellulose acetate with a $D S$ less than 2.4, probably because of its lesser industrial importance.

In order to understand the fundamental properties of their cellulose derivatives, it is important to investigate their solution properties over a wide range of $D S$. Cellulose acetate, of course, is not an exception. In this study, we synthesized watersoluble incompletely substituted cellulose acetate $(D S=0.49)$ and performed solution viscosity, membrane osmometry, and light scattering measurements in a variety of solvents. The effect of $D S$ and the solvent nature on the molecular charac- 
teristics of cellulose acetate is discussed.

\section{EXPERIMENTAL}

\section{Preparation of Water-Soluble Cellulose Acetate}

Cellulose diacetate flakes $(D S=2.46$, weightaverage molecular weight $M_{w}=1.2 \times 10^{5}$ ) synthesized in a previous paper ${ }^{5}$ was crushed into a fine powder in a ball-mill and hydrolyzed using hydrochloric acid as catalyst, in a $77 \%$ aq acetic acid solution under nitrogen atomosphere at $40^{\circ} \mathrm{C}$ for various times of period. The incompletely substituted cellulose acetate thus hydrolyzed was recovered by precipitation with acetone, filtered, washed with acetone to remove all traces of acetic acid in the polymer, dryed in vacuo at room temperature and finally stocked in a dark, cold place. Ten cellulose acetate whole polymers of various $D S$ (polymer code MAW-1-MAW-10) were prepared in this manner.

The combined acetic acid content $(A C)$ of the unfractionated and fractionated cellulose acetates was determined by back-titration, using sodium hydroxide and sulfulic acid. From $\mathrm{C}^{13}$-NMR analysis of the CA whole polymer (sample code MAW$5, D S=0.49$ ) dissolved in deuterated dimethyl sulfoxide (DMSO- $d_{6}$ ), the probability of finding $O$ acetyl groups attached to the $\mathrm{C}_{2}, \mathrm{C}_{3}$, and $\mathrm{C}_{6}$ atoms was estimated to be $0.10,0.20$, and 0.19 , respectively. ${ }^{9}$

\section{Molecular Weight Fractionation}

The successive solutional fractionation (SSF) technique was applied. The original polymer (polymer code MAW-5; $A C=16.1 \%, D S=0.49$ ) in water was fractionated at $25^{\circ} \mathrm{C}$, using methanol, into 15 approximately equal fractions. The polymer volume fraction $v_{\mathrm{p}}{ }^{0}$ in the first fractionation step, from which a phase separation occurred, was $0.56 \%$. In order to lessen the time necessary for the precipitate to settle completely in the bottom of the fractionation vessel, a small amount of sodium chloride was added to the aqueous solution. The supernatant phase was concentrated by evaporating off the water and methanol at $60^{\circ} \mathrm{C}$, leaving a dry powder which was dissolved in water to make up a solution of $5 \mathrm{wt} \%$. A large quantity of acetone was then added to completely precipitate the fraction, which was then filtered, washed with acetone and dried in vacuo.

\section{Solution Viscosity}

Solution viscosities of CA $(D S=0.49)$ fractions were measured in $N, N$-dimethylacetamide (DMAc), DMSO, water, and formamide (FA) using a modified Ubbelohde suspension-type viscometer at $25^{\circ} \mathrm{C}$. The limiting viscosity number $[\eta]$ was determined from reduced viscosities $\eta_{\mathrm{sp}} / c\left(\eta_{\mathrm{sp}}\right.$, specific viscosity; $c$, polymer mass concentration) at five concentrations by extrapolation to infinite dilution.

\section{Membrane Osmometry}

Osmotic measurements were carried out on solutions of CA in DMAc at $25^{\circ} \mathrm{C}$ with a HewlettPackard high-speed membrane osmometer model 502. The membrane was the Ultracella filter-type SH-11539 (regenerated cellulose, allerfeinst) manufactured by Sartorius-Membrane filter GMBH (West Germany).

\section{Light Scattering}

DMAc, water and FA were chosen as the solvents for the light-scattering measurements. CA $(D S=$ $0.49)$ fractions were dissolved in freshly distilled solvents at $60^{\circ} \mathrm{C}$ to make $0.6 \mathrm{wt} \%$ solutions. The solutions were left standing for at least more than $16 \mathrm{~h}$ and then filtered with a sintered glass filter ( 2 nd grade). The specific refractive index increments $\partial n / \partial c$ were determined by a Shimadzu differential refractiometer, model DR- 4 at $25^{\circ} \mathrm{C}$ at a wavelength $\lambda_{0}$ of $436 \mathrm{~nm}$ to be $0.068,0.131$, and $0.069\left(\mathrm{~cm}^{3} \mathrm{~g}^{-1}\right)$ for CA fractions in DMAc, water, and FA, respectively. The stock solutions were centrifuged at $1.03 \times 10^{5} \mathrm{~g}$ for $2 \mathrm{~h}$ in a Hitachi model $55 \mathrm{p}-7$ automatic preparative ultracentrifuge. The upper twothirds of the supernatant phase was carefully filtered through a Sartorius membrane filter $0.15 \mu \mathrm{m}$ and directly transferred into the light-scattering cylindrical cell. The light-scattering intensity was measured within an angle range from $30-150^{\circ}$ using unpolarized incident light $\lambda_{0}=436 \mathrm{~nm}$ at $25^{\circ} \mathrm{C}$ in a FICA model 42000 photogonio diffusiometer. The data obtained were analyzed according to Zimm's procedure. ${ }^{10,11}$

\section{DISCUSSION}

\section{Solubility Behavior}

Ten cellulose acetate samples with $D S$ ranging from 0.10 to 1.96 were used in the water solubility test. Table I shows the results from this test. CA is 
Dilute Solution Properties of Water-Soluble CA

Table I. Degree of substitution $(D S)$ dependence on water solubility of cellulose acetate ${ }^{\mathrm{a}}$

\begin{tabular}{lccccccccccc}
\hline Polymer & MAW-1 & MAW-2 & MAW-3 & MAW-4 & MAW-5 & MAW-6 & MAW-7 & MAW-8 & MAW-9 & MAW-10 \\
\hline DS & 1.96 & 0.98 & 0.81 & 0.65 & 0.49 & 0.39 & 0.34 & 0.33 & 0.16 & 0.10 \\
Solubility & $\times$ & $\triangle$ & $\bigcirc$ & $\bigcirc$ & 0 & $\bigcirc$ & $\triangle$ & $\triangle$ & $\times$ & $\times$ \\
\hline
\end{tabular}

a $\bigcirc$, soluble; $\triangle$, only swell; $\times$, insoluble.

water-soluble in a $D S$ range of $0.39-0.81$. It has for years been believed that CA is soluble in water in a $D S$ range $0.6-0.8$ at room temperature. ${ }^{12} \mathrm{But}, \mathrm{CA}$ with a $D S$ lower than 0.6 (in this case, 0.4) is evidently soluble in water at room temperature. The CA sample (polymer code MAW-5, $D S=0.49$ ) was used for a further study.

In order to confirm the stability of aq CA solution, a $0.6 \mathrm{wt} \%$ solution of the $\mathrm{CA}$ whole polymer $(D S=0.49)$ in water was prepared and stocked in a dark place at $25^{\circ} \mathrm{C}$. Figure 1 seems to indicate that although water is a poor solvent in light scattering measurements (see Table III) and CA molecules have a rather high tendency to crystallize when coagulated or precipitated as gels on heattreatment, an aq solution of CA $(D S=0.49)$ is stable at least for several months at room temperature, with no indication of crystallization.

The solubility behavior of the CA whole polymer $(D S=0.49)$ in various organic solvents was exam- ined. The polymer is found to be insoluble in less polar solvents such as hydrocarbons, chlorinated hydrocarbons, amines, esters, but soluble in very polar organic solvents such as formic acid (dielectric constant $\left.\varepsilon=58.5^{13}\right)$, trifluoroacetic acid $\left(\varepsilon=39.5^{13}\right)$, dimethylacetamide $\left(\varepsilon=37.8^{13}\right)$, dimethyl sulfoxide $\left(\varepsilon=46.6^{13}\right)$, water $\left(\varepsilon=78.3^{13}\right)$, and formamide

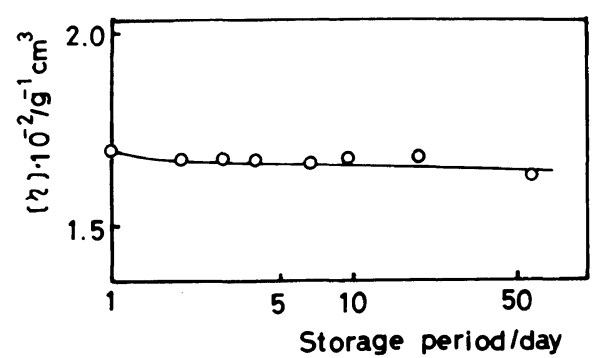

Figure 1. Limiting viscosity number $[\eta]$ of cellulose acetate MAW-5 in water as a function of storage period of the original solution $(0.6 \mathrm{wt} \%)$ at $25^{\circ} \mathrm{C}$.

Table II. Results of successive fractionation run (water-methanol) and viscosity measurements on cellulose acetate $(D S=0.49)$ in various solvents

\begin{tabular}{|c|c|c|c|c|c|c|c|c|c|c|}
\hline \multirow{2}{*}{$\begin{array}{l}\text { Sample } \\
\text { code }\end{array}$} & \multirow{2}{*}{$v_{\mathrm{m}}$} & \multirow{2}{*}{$\rho_{\mathrm{s}}$} & \multicolumn{4}{|c|}{$[\eta] / \mathrm{cm}^{3} \mathrm{~g}^{-1}\left(\right.$ at $\left.25^{\circ} \mathrm{C}\right)$} & \multicolumn{4}{|c|}{ Huggins coefficient $k^{\prime}$} \\
\hline & & & DMAc & DMSO & $\mathrm{H}_{2} \mathrm{O}$ & FA & DMAc & DMSO & $\mathrm{H}_{2} \mathrm{O}$ & FA \\
\hline MA-1 & 0.800 & 0.102 & 42 & 38 & 40 & - & 0.45 & - & 0.52 & - \\
\hline MA-2 & 0.755 & 0.085 & - & 79 & 80 & 87 & - & 0.39 & 0.62 & 0.46 \\
\hline MA-3 & 0.730 & 0.120 & $108^{\circ}$ & 106 & 118 & 124 & 0.43 & 0.53 & 0.52 & 0.49 \\
\hline MA-4 & 0.720 & 0.111 & 142 & 140 & 157 & - & 0.43 & 0.42 & 0.52 & - \\
\hline MA-5 & 0.715 & 0.044 & 133 & 132 & 151 & 156 & 0.38 & 0.45 & 0.47 & 0.57 \\
\hline MA-6 & 0.710 & 0.056 & 154 & 153 & 172 & 180 & 0.40 & 0.41 & 0.52 & 0.42 \\
\hline MA-7 & 0.707 & 0.027 & 167 & 167 & 172 & 180 & 0.34 & 0.39 & 0.52 & 0.49 \\
\hline MA-8 & 0.703 & 0.032 & 164 & 162 & 182 & 188 & 0.43 & 0.47 & 0.60 & 0.49 \\
\hline MA-9 & 0.699 & 0.020 & 185 & 178 & 193 & 198 & 0.35 & 0.40 & 0.59 & 0.60 \\
\hline MA- 10 & 0.695 & 0.034 & 189 & 201 & 203 & 220 & 0.44 & 0.44 & 0.67 & 0.62 \\
\hline MA-11 & 0.691 & 0.018 & 200 & - & 215 & 230 & 0.48 & - & 0.69 & 0.68 \\
\hline MA-12 & 0.682 & 0.033 & 203 & 204 & 232 & 232 & 0.48 & 0.49 & 0.64 & 0.74 \\
\hline MA-13 & 0.671 & 0.054 & 235 & 235 & 259 & 257 & 0.57 & 0.56 & 0.78 & 0.74 \\
\hline MA-14 & 0.660 & 0.111 & - & - & 234 & - & - & - & - & - \\
\hline MAW-5 & - & 0.847 & 170 & 170 & 170 & - & 0.35 & 0.35 & 0.65 & - \\
\hline CMA-W1 & - & - & 452 & - & - & - & 0.46 & - & - & - \\
\hline
\end{tabular}


$\left(\varepsilon=111^{13}\right)$. It should be noted that the solubility of CA $(D S=0.49)$ is controlled primarily by the dielectric constant of the solvent and the critical value of this constant above which the polymer dissolves is approximately 37 .

\section{Fractionation of $C A(D S=0.49)$}

The second and third columns of Table II give the composition of the methanol mixture, $v_{\mathrm{m}}$, for each fractionation step and the fraction size $\rho_{\mathrm{s}}$. $v_{\mathrm{m}}$ varies from 0.80 to 0.66 . The total recovery of the polymer was $85 \%$. The fourth to eleventh columns in the same table compile the limiting viscosity numbers $[\eta]$ and Huggins coefficients $k^{\prime}$ for CA in DMAc, DMSO, water, and FA. [ $\eta]$ of the fractions in water covers a range from 40 to 259 , indicating that fractionation according to molecular weight was performed successfully. The $D S$ values for all the fractions are within $0.49 \pm 0.01$ and thus can be regarded as independent of $M_{w}$.

\section{Molecular Weight and Molecular Weight Distribution of the Fractions \\ The Zimm plots for the CA $(D S=0.49)$ fraction} MA-5 in DMAc and formamide are shown in Figures 2 and 3. No downward curvature can be seen in these plots.

Tables III and IV summarize the experimental results from light scattering (LS) and membrane osmometry $(\mathrm{MO})$ on CA $(D S=0.49)$ solutions in DMAc, water, and formamide at $25^{\circ} \mathrm{C}$. The maximum value of $M_{w}$ for these fractions is a little below $15 \times 10^{4}$, which is an upper limit as long as the hydrolysis method is employed and a present there is no efficient method for preparing CA $(D S \leqq 0.6)$ with a higher $M_{w}$.

Excellent agreement of the $M_{w}$ values of the fraction MA-5 in three different solvents confirms definitely the experimental reliability and also indicates that the CA $(D S=0.49)$ molecules dissolve molecularly in these solvents without association. The experimental error involved in the lightscattering measurement of $M_{w}$ was estimated to be less than a few $\%$.

The polydispersity parameter $M_{w} / M_{n}$, as determined by LS and MO, $1.30 \pm 0.02$ for the series of CA $(D S=0.49)$ fractions was used here.

The weight-average radius of gyration $\left\langle S^{2}\right\rangle_{w}^{1 / 2}$ was calculated from the $z$-average radius of gyration $\left\langle S^{2}\right\rangle_{z}^{1 / 2}$ and the $M_{w} / M_{n}$ value, assuming the Schulz-

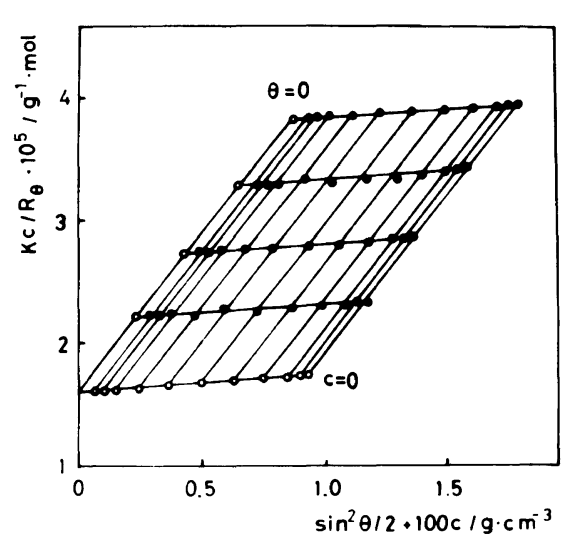

Figure 2. Zimm plot of a cellulose acetate $(D S=0.49)$ fraction MA-5 in DMAc at $25^{\circ} \mathrm{C}$ : unpolarized incident light wave length $\lambda_{0}=436 \mathrm{~nm}$; closed circles, measured points; open circles, points obtained by extrapolation.

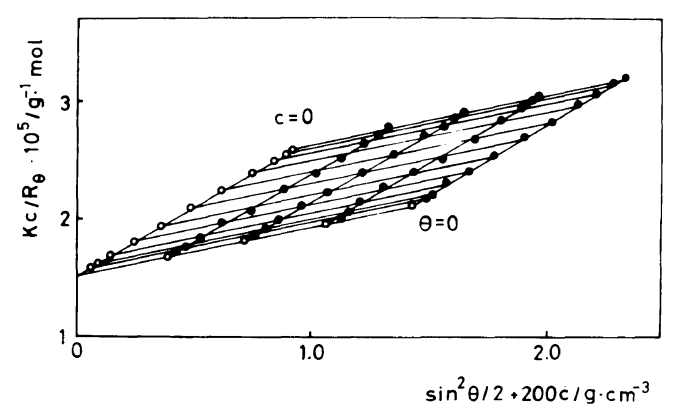

Figure 3. Zimm plot of a cellulose $(D S=0.49)$ fraction MA- 5 in formamide at $25^{\circ} \mathrm{C}$ : unpolarized incident light wave length $\lambda_{0}=436 \mathrm{~nm}$.

Zimm molecular weight distribution. ${ }^{6}$

\section{Mark-Houwink-Sakurada Equations}

Figures $4 \mathrm{a}-\mathrm{d}$ show the log-log plots of $[\eta] v s . M_{w}$ for CA $(D S=0.49)$ in DMAc, DMSO, water, and formamide at $25^{\circ} \mathrm{C}$ (open circles). From this figure the Mark-Houwink-Sakurada (MHS) equation,

$$
[\eta]=K_{\mathrm{m}} M_{w}{ }^{a}
$$

can be established by using the least-squares method. The results are as follows:

$$
\begin{aligned}
& {[\eta]=0.19_{1} M_{w}^{0.60} \text { in DMAc }} \\
& {[\eta]=0.17_{1} M_{w}^{0.61} \text { in DMSO }} \\
& {[\eta]=0.20_{9} M_{w}^{0.60} \quad \text { in water }}
\end{aligned}
$$

and 
Table III. Results of light-scattering, membrane-osmometry, and viscosity measurements on cellulose acetate $(D S=0.49)$ in dimethylacetamide at $25^{\circ} \mathrm{C}$

\begin{tabular}{|c|c|c|c|c|c|c|c|c|c|}
\hline \multirow{3}{*}{$\begin{array}{c}\text { Sample } \\
\text { code }\end{array}$} & \multicolumn{4}{|c|}{ Light scattering } & \multirow{3}{*}{$\begin{array}{l}\begin{array}{l}\text { Osmotic } \\
\text { pressure }\end{array} \\
M_{n} \times 10^{-4}\end{array}$} & \multirow{3}{*}{$M_{w} / M_{n}$} & \multirow{3}{*}{$\Phi \times 10^{-23}$} & \multirow{3}{*}{$\alpha_{\mathrm{s}}^{\mathrm{b}}$} & \multirow{3}{*}{$X^{\mathrm{c}}$} \\
\hline & \multirow{2}{*}{$M_{w} \times 10^{-4}$} & $\left\langle S^{2}\right\rangle_{z}^{1 / 2} \times 10^{8}$ & \multicolumn{2}{|c|}{$\left\langle S^{2}\right\rangle_{w}^{1 / 2} \times 10^{8} \quad A_{2} \times 10^{4}$} & & & & & \\
\hline & & $\mathrm{cm}$ & $\mathrm{cm}$ & $\mathrm{cm}^{3} \mathrm{~mol} \mathrm{~g}^{-2}$ & & & & & \\
\hline MA-1 & - & - & - & - & 0.97 & - & - & - & - \\
\hline MA-2 & - & - & - & - & 1.67 & - & - & - & \\
\hline MA-3 & 4.55 & 137 & 123 & 4.80 & - & - & 1.85 & 1.03 & 4.3 \\
\hline MA-4 & 6.25 & 160 & 144 & 8.90 & - & - & 2.08 & 1.07 & 8.0 \\
\hline MA-5 & 6.32 & 164 & 148 & 7.50 & - & - & 1.82 & 1.05 & 4.2 \\
\hline MA-6 & 6.76 & 175 & 158 & 14.9 & 5.20 & 1.30 & 1.86 & 1.18 & 6.2 \\
\hline MA-7 & 7.25 & 184 & 166 & 13.8 & - & - & 1.86 & 1.18 & 6.2 \\
\hline MA-8 & 7.94 & 189 & 170 & 15.1 & 6.10 & 1.31 & 1.85 & 1.26 & 7.8 \\
\hline MA-9 & 8.00 & 191 & 172 & 15.0 & - & - & 2.01 & 1.24 & 10.5 \\
\hline MA-10 & 10.8 & 215 & 194 & 12.1 & - & - & 1.96 & 1.26 & 10.1 \\
\hline MA-11 & 11.1 & 223 & 201 & 10.2 & - & - & 1.92 & 1.13 & 6.6 \\
\hline MA-12 & 11.3 & - & - & - & 8.70 & 1.30 & - & - & - \\
\hline MA-13 & 14.5 & 253 & 223 & 3.1 & 11.3 & 1.28 & 2.18 & 1.03 & 9.0 \\
\hline MAW $-5^{\mathrm{a}}$ & 8.00 & 247 & 198 & 13.8 & 3.90 & 2.29 & - & - & - \\
\hline
\end{tabular}

Table IV. Results of solution-viscosity and light-scattering measurements on cellulose acetate $(D S=0.49)$ fraction MA-5 in formamide, water, and DMAc at $25^{\circ} \mathrm{C}$

\begin{tabular}{|c|c|c|c|c|c|c|c|c|c|}
\hline \multirow{2}{*}{ Solvent } & \multirow{2}{*}{$\begin{array}{c}\text { Dielectric } \\
\text { constant } \\
\varepsilon\end{array}$} & \multirow{2}{*}{$\frac{[\eta]^{\mathrm{a}}}{\mathrm{cm}^{3} \mathrm{~g}^{-1}}$} & \multirow{2}{*}{$M_{w} \times 10^{-4}$} & \multirow{2}{*}{$\frac{\left\langle S^{2}\right\rangle_{z}^{1 / 2} \times 10^{8}}{\mathrm{~cm}}$} & \multirow{2}{*}{$\frac{A_{2} \times 10^{4}}{\mathrm{~cm}^{3} \mathrm{~mol} \mathrm{~g}^{-2}}$} & \multirow{2}{*}{$\Phi \times 10^{-23}$} & \multirow{2}{*}{$\Psi \times 10^{3}$} & \multirow{2}{*}{$\alpha_{\mathrm{s}}$} & \multirow{2}{*}{$X$} \\
\hline & & & & & & & & & \\
\hline FA & 111 & 156 & 6.30 & 349 & 8.4 & 0.22 & 8.7 & 1.01 & 0.14 \\
\hline Water & 78.3 & 151 & 6.42 & 291 & 3.1 & 0.38 & 6.7 & 1.01 & 0.26 \\
\hline DMAc & 37.8 & 133 & 6.32 & 164 & 7.5 & 1.82 & 4.9 & 1.05 & 4.2 \\
\hline
\end{tabular}

$$
[\eta]=0.20_{9} M_{w}^{0.60} \quad \text { in } \quad \text { FA }
$$

The molecular weight range covered by eq $\mathrm{a}-\mathrm{d}$ is $(4.55-14.5) \times 10^{4}$ (the ratio of the maximum to minimum $M_{w}$ is 3.2). The MHS equations in water and in formamide coincide and the values for exponent $a$ in the above equations range from 0.60 to 0.61 in these different solvents $(\varepsilon=37.8 \sim 111)$. Thus, $a$ is very insensitive to the solvent.

Equation a should be compared with those established for CA $(D S=2.92)$ and CA $(D S=2.46)$ in the same solvent at the same temperature. ${ }^{5}$

$$
[\eta]=0.026_{4} M_{w}^{0.75} \text { for } \mathrm{CA}(D S=2.92)
$$

$$
[\eta]=0.039_{5} M_{w}^{0.74} \text { for } \quad \mathrm{CA}(D S=2.46)
$$

In Figure $4 \mathrm{a}$, open rectangles represent $\mathrm{CA}$ $(D S=2.92)$ and the open triangles stand for CA $(D S=2.46)$, as reported in a previous paper. ${ }^{6}$ In the lower $M_{w}$ range, $[\eta]$ of CA $(D S=0.49)$ is similar to that of CA $(D S=2.46)$, but in the higher $M_{w}$ range, $[\eta]$ for CA $(D S=0.49)$ approaches that for CA $(D S=2.92)$. The exponent $a$ for CA $(D S=0.49)$ is substantially smaller than the values obtained for CA $(D S=2.46$ and 2.92) in DMAc.

The limiting viscosity number $[\eta]$ of $\mathrm{CA}$ is a unique function of the dielectric constant $\varepsilon$ of the solvent in which the polymer is dissolved. Figure 5 is 


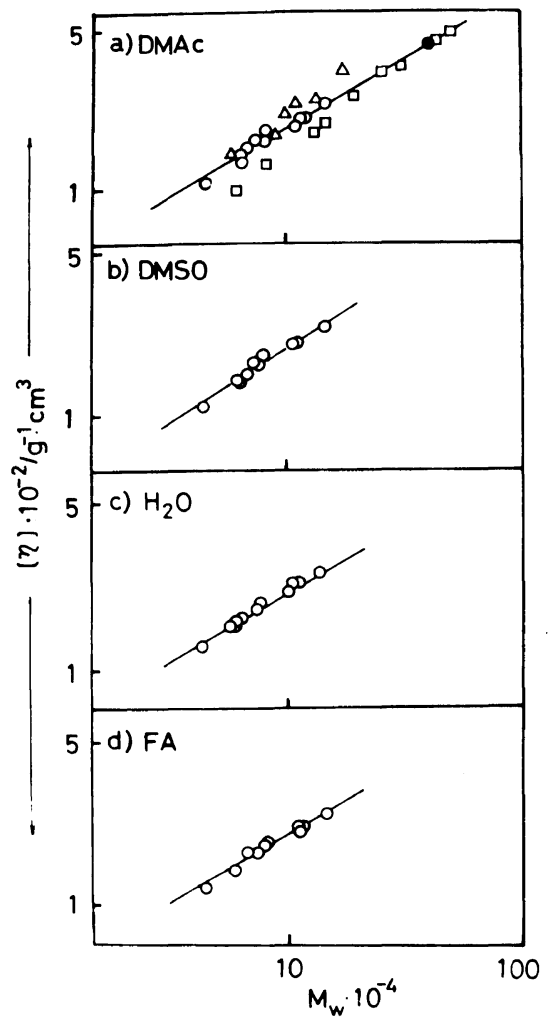

Figure 4. Log-log plot of limiting viscosity number $[\eta]$ against the weight-average molecular weight $M_{w}$ for cellulose acetate $(D S=0.49)$ in dimethylacetamide (DMAc), dimethyl sulfoxide (DMSO), water, and formamide at $25^{\circ} \mathrm{C}$. Straight lines represent the MarkHouwink-Sakurada equations (eq a-d) determined by the least-squares method: $O, \mathrm{CA}(D S=0.49)$ (this work); $\triangle$, CA $(D S=2.46)^{5} ; \square, \mathrm{CA}(D S=2.92){ }^{6}$

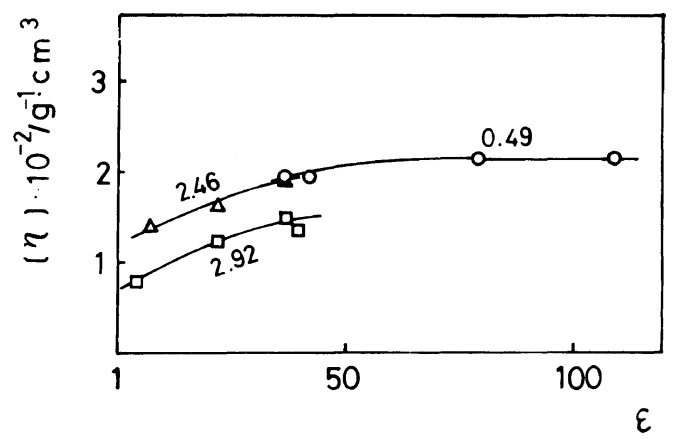

Figure 5. Limiting Viscosity number $[\eta]$ of three CA polymers having the dielectric constant $\varepsilon$ of the solvent in which the polymers were dissolved: $\bigcirc, \mathrm{CA}$ $(D S=0.49) ; \triangle$, CA $(D S=2.46)^{5,6} ; \square, \mathrm{CA}(D S=2.92)^{6}$

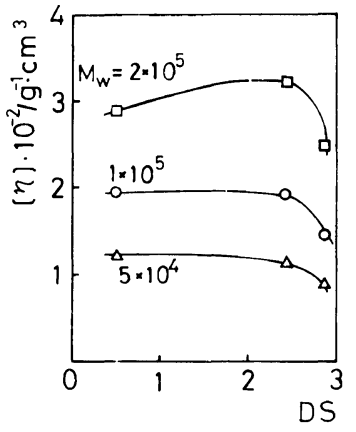

Figure 6. Effect of the degree of substitution $(D S)$ on limiting viscosity number $[\eta]$ of cellulose acetate in DMAc at $25^{\circ} \mathrm{C}$.

an example in which three kinds of CA fractions with the same molecular weight $\left(M_{w}=1 \times 10^{5}\right)$ and various $D S(0.49,2.46,2.92)$ are shown. $[\eta]$ increases with an increase in $\varepsilon$, approaching an asymptotic value which depends on the $D S$ value of the polymer. This phenomenon can not be interpreted in terms of the excluded volume effect (see, Table IV), but may be attributed to a significant change in the unperturbed chain dimensions with the solvent nature. Recently, we confirmed by nuclear magnetic resonance (NMR) that there exists a specific strong interaction between the $O$-acetyl proton or the hydroxyl proton of the CA molecule and the solvent and that the strength of this interaction depends on. the solvent nature. ${ }^{14}$

The effect of the degree of substitution $(D S)$ on the limiting viscosity number $[\eta]$ of CA in DMAc is illustrated in Figure 6, in which the value for CA $(\dot{D} S=0.49)$ with $M_{w}=2 \times 10^{5}$ is calculated from eq a. Other points for CA $(D S=2.46)$ and $\mathrm{CA}$ $(D S=2.92)$ are calculated from eq $\mathrm{d}$ and a in ref 6 . The $D S$ dependence of $[\eta]$ varies with $M_{w}$ of the polymer; for relatively small $M_{w},[\eta]$ decreases monotonously with an increase in $D S$, but for large $M_{w}\left(M_{w} \gtrsim 2 \times 10^{5}\right),[\eta]$ reveals a maximum at a $D S$ value. In the $D S$ range of $0.49-2.46$, $[\eta]$ is not very sensitive to $D S$, slightly increasing or decreasing as $D S$ gets large. In the comparatively narrow $D S$ range from $2.46-2.92,[\eta]$ decreases remarkably with an increase in $D S$. This seems to indicate that the interaction between the hydroxyl groups remaining on the CA molecule and the solvent plays an important role in the solution viscosity. 
Radius of gyration and Second Virial Coefficient

The molecular weight dependence of the $z$ average radius of gyration $\left\langle S^{2}\right\rangle_{z}^{1 / 2}$ in DMAc is shown in Figure 7 for CA $(D S=0.49)$ in DMAc by open circles. In this figure, the data points for CA $(D S=2.92)$ and CA $(D S=2.46)$ are also included as open rectangles and an open triangle for comparison. Among these three kinds of $\mathrm{CA}$ with different $D S$, CA $(D S=0.49)$ has the most compact form in DMAc.

$$
\begin{aligned}
& \left\langle S^{2}\right\rangle_{z}^{1 / 2} \text { is related to } M_{w} \text { by } \\
& \left\langle S^{2}\right\rangle_{z}^{1 / 2}=0.50 \times 10^{-8} M_{w}^{0.52} \quad(\mathrm{~cm})
\end{aligned}
$$

for $\mathrm{CA}(D S=0.49)$ in DMAc at $25^{\circ} \mathrm{C}$

It is interesting that $\left\langle S^{2}\right\rangle_{z}^{1 / 2}$ of the $\mathrm{CA}(D S=0.49)$ fraction in a given solution (closed circle in Figure 7) is approximately twice the value in DMAc. The CA $(D S=0.49)$ polymer expands in the following order: DMAc $<$ water $<$ formamide.

The second virial coefficient $A_{2}$, determined for $\mathrm{CA}(D S=0.49)$ in DMAc at $25^{\circ} \mathrm{C}$ by membrane osmometry and light scattering shows that there is too much scatter around $1.1 \times 10^{-3}\left(\mathrm{~cm}^{3} \mathrm{~g}^{-2} \mathrm{~mol}\right)$ to allow a definite conclusion as to its molecular weight dependence.

The $A_{2}$ values of the CA $(D S=0.49)$ fraction for the three solvents are of the order of $10^{-4} \mathrm{~cm}^{3}$ molg ${ }^{-2}$, thus showing no systematic variation in the solvent (see the sixth column of Table IV).

Flory's viscosity parameter

$$
\begin{aligned}
\Phi[ & \equiv[\eta] M_{w} /\left(q_{w, z}^{-1} 6^{3 / 2}\left\langle S^{2}\right\rangle_{z}^{3 / 2}\right), q_{w, z} \\
& =\text { correction factor for polydispersity }]
\end{aligned}
$$

for CA $(D S=0.49)$ in DMAc is plotted against $M_{w}$ as open circles in Figure 8. The corresponding values for aqueous and formamide solutions are shown in the seventh column of Table IV. Here, $q_{w, z}(=1.41)$ was calculated assuming the Schulz-Zimm distribution. The $\Phi$ for DMAc solution is smaller than that for CA $(D S=2.92)$, in the same solvent (as denoted by the open rectangles), being 1.3-1.6 times smaller than the theoretical value $\left(2.87 \times 10^{23}\right)$ for a non-permeable polymer chain in the unperturbed state. The $\Phi$ values for CA $(D S=0.49)$ in DMAc shows a significant molecular weight dependence which may be expressed by,

$$
\Phi=K_{\Phi} M_{\Phi}{ }^{a}
$$

where $K_{\Phi}$ and $a_{\Phi}$ are the parameters for a given

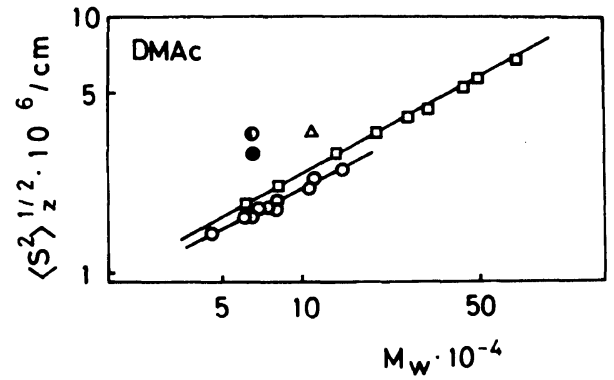

Figure 7. Molecular weight dependence of the radii of gyration $\left\langle S^{2}\right\rangle_{z}^{1 / 2}$ for cellulose acetate in DMAc at $25^{\circ} \mathrm{C}$ : $\bigcirc, \mathrm{CA}(D S=0.49)$ (this work); $\triangle, \mathrm{CA}(D S=2.46)^{5} ; \square$, CA $(D S=2.92)^{6} ; 0$, CA $(D S=0.49)$ in water; $\mathbf{O}, \mathrm{CA}$ $(D S=0.49)$ in formamide.

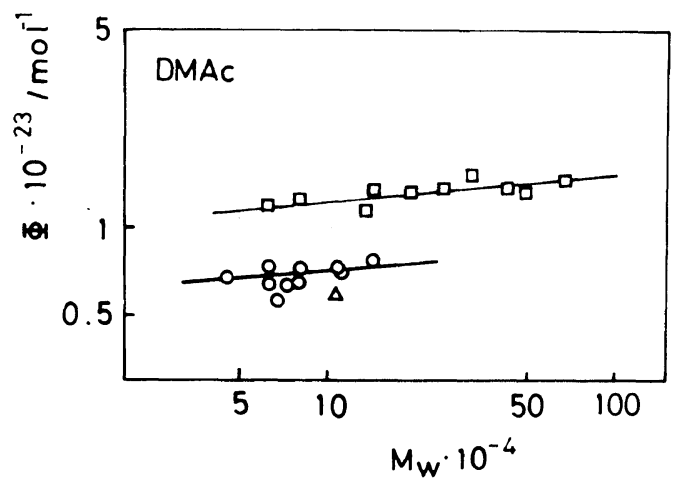

Figure 8. Molecular weight dependence of the Flory's viscosity parameter $\Phi$ for cellulose acetate in DMAc at $25^{\circ} \mathrm{C}: \quad \bigcirc$, CA $(D S=0.49) \quad$ (this work); $\triangle$, CA $(D S=2.46)^{5} ; \square$, CA $(D S=2.92)^{6}$.

polymer-solvent system. For CA $(D S=0.49)$ in DMAc, we obtain $K_{\Phi}=0.61 \times 10^{23}$ and $a_{\Phi}=0.103$, which due to a relatively narrow range of $M_{w}$, are not accurate enough to estimating the unperturbed chain dimensions. The open triangle in Figure 8 denotes CA $(D S=2.46)$ in DMAc.

The $\Phi$ value for CA in DMAc decrease in the order: CA $(D S=2.92)>\mathrm{CA}(D S=0.49)>\mathrm{CA}$ $(D S=2.46)$. The $\Phi$ values for CA $(D S=0.49)$ in water and formamide are unexpectedly small, being approximately $1 / 16$ and $1 / 30$ the value in DMAc. This seems attributable to the large $\left\langle S^{2}\right\rangle_{z}^{1 / 2}$ of CA in these solvents. Abnormally small values of $\Phi$ and its molecular dependence for cellulose acetate $(D S=0.49-2.92)$ undoubtedly indicate a draining effect. 
The expansion factor $\alpha_{\mathrm{s}}$ in these solutions was calculated, according to the procedure described previously, ${ }^{15}$ using the penetration function $\Psi\left(\equiv\left(4 \pi^{3 / 2} N_{\mathrm{a}}\right)^{-1}\left(A_{2} M^{2} /\left\langle S^{2}\right\rangle^{3 / 2}\right)\right.$. The results are listed in the ninth column of Table III and in the eighth column of Table IV. The $\alpha_{\mathrm{s}}$ values obtained remain almost constant within experimental error, and are close to unity, suggesting that all the solvents exployed are thermodynamically poor solvents for CA. It should be remembered that any theory yields nearly the same relations between $\Psi$ and the excluded volume parameter $\bar{z}$ for $\Psi \leqq 0.1$ which is the range in the CA $(D S=0.49)$-solvent systems (the eighth column of Table IV). Using $\Phi$ and $\alpha_{\mathrm{s}}$, the draining parameter $X$, defined in the Kurata-Yamakawa theory, ${ }^{16}$ was estimated (this method is referred to as the method $1 \mathrm{~A}$ in the previous paper ${ }^{15}$ ), and is given in the last columns of Table III and IV. For the CA $(D S=0.49)$-DMAc system, the $X$ values obtained in this narrow molecular weight range are too small to permit examination of their molecular dependence. These values were found to be from 4.2 to 10.5 (average 7.3), and are smaller than $X \gtrsim 10$ is the case for many vinyltype polymers.

Figure 9 shows the variation in $[\eta],\left\langle S^{2}\right\rangle_{z}^{1 / 2}, \Phi, \alpha_{\mathrm{s}}$, and $X$ for the CA $(D S=0.49)$ fraction $\left(M_{w}=6.32 \times 10^{4}\right)$ with the dielectric constant $\varepsilon$ of the solvent. Evidently, an increase in $\varepsilon$ leads to larger values of $[\eta]$ and $\left\langle S^{2}\right\rangle_{z}^{1 / 2}$ and smaller values of $\Phi$ and $X . \alpha_{\mathrm{s}}$ is nearly independent of $\varepsilon$. This strongly suggests that at least the observed marked variation in $[\eta],\left\langle S^{2}\right\rangle_{z}^{1 / 2}$ and $\Phi$ does not arise from variation in the long-range interaction parameter.

\section{Unperturbed Chain Dimension}

The unperturbed chain dimension $A$ is defined by,

$$
A=\left(\left\langle R^{2}\right\rangle_{0} / M\right)^{1 / 2}
$$

with,

$$
\left\langle R^{2}\right\rangle_{0}=6\left\langle S^{2}\right\rangle_{0}
$$

$\left(\left\langle R^{2}\right\rangle_{0}\right.$ is the mean-square end-to-end distance of the polymer chain in the unperturbed state) was estimated by using the methods $2 \mathrm{~B}, 2 \mathrm{C} 2 \mathrm{E}$, and $2 \mathrm{~F}$, proposed in the previous paper. ${ }^{15}$

Method 2B:

$$
\left\langle S^{2}\right\rangle_{0}^{1 / 2}=\left\langle S^{2}\right\rangle^{1 / 2} / \alpha_{\mathrm{s}}
$$

Method 2C: Baumann plot ${ }^{17}$

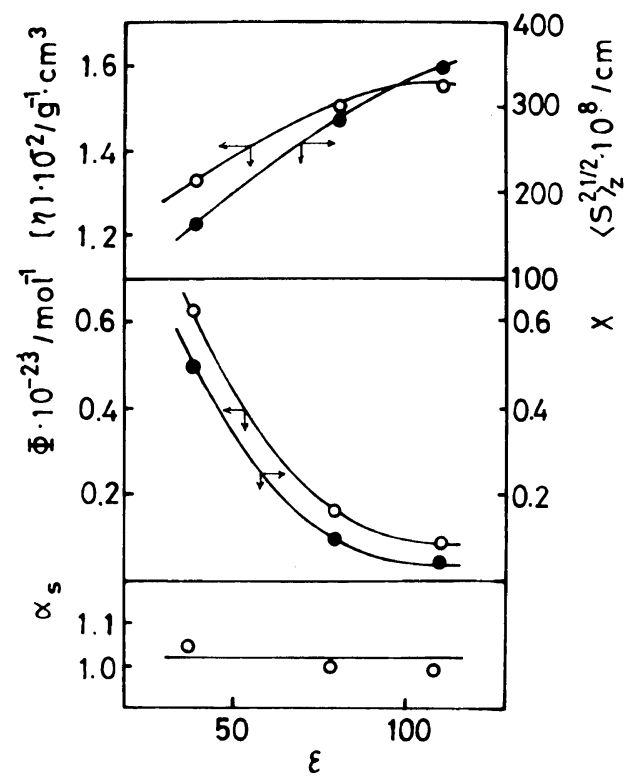

Figure 9. Dependence of limiting viscosity number $[\eta]$, radius of gyration $\left\langle S^{2}\right\rangle_{z}^{1 / 2}$, Flory's viscosity parameter $\Phi$, expansion factor $\alpha_{\mathrm{s}}$ and draining parameter $X$ of CA $(D S=0.49)$ fraction $\mathrm{MW}-5$ on the dielectric constant of the solvent.

$$
\left\langle S^{2}\right\rangle^{3 / 2} / M^{3 / 2}=A^{3} / 6^{3 / 2}+\left(1 / 4 \pi^{3 / 2}\right) B M^{1 / 2}
$$

Method 2E: Stockmayer-Fixman plot ${ }^{18}$

$$
[\eta] / M^{1 / 2}=K+2(3 / 2)^{3 / 2} \Phi_{0}(\infty) B M^{1 / 2}
$$

with

$$
K=\Phi_{0}\left(X_{0}\right) A^{3}
$$

Method 2F: Kamide et al. plot $^{19-24}$

$$
\begin{aligned}
& -\log K_{\mathrm{m}}+\log \left[1+2\left\{(a-0.5)^{-1}-2\right\}^{-1}\right] \\
& =-\log K+(a-0.5) \log M_{0}
\end{aligned}
$$

$\Phi_{0}(X)$ denotes $\Phi(X)$ at $\alpha_{\mathrm{s}}=1$, which is a function of the draining parameter $X$, and $M_{0}$ is the geometric mean of the upper and lower molecular weight limit for which eq.1 holds.

In Figure 10, the ratio $\left\langle S^{2}\right\rangle_{0, w} / M$ evaluated by the method 2B is plotted against $M_{w}$ for CA $(D S=0.49)$ in DMAc at $25^{\circ} \mathrm{C}$. The plotted points scatter considerably around $\left\langle S^{2}\right\rangle_{0, w} / M_{w}=0.27 \times 10^{-6} \mathrm{~cm}^{2} \mathrm{~g}^{-1}$ mol (accordingly, $A\left(\equiv\left(6\left\langle S^{2}\right\rangle_{0} / M^{1 / 2}\right)=1.28 \times 10^{-8}\right.$ $\mathrm{cm})$ and the ratio $\left\langle S^{2}\right\rangle_{0, w} / M_{w}$ can be regarded as approximately constant (i.e., $a_{2} \equiv \mathrm{d} \ln \left\langle S^{2}\right\rangle_{0, w} /$ $\left.M / \mathrm{d} \ln M_{w} \simeq 0\right)$. The CA $(D S=0.49)$ chain in the unperturbed state can be considered Gaussian to a first approximation. The same method was 


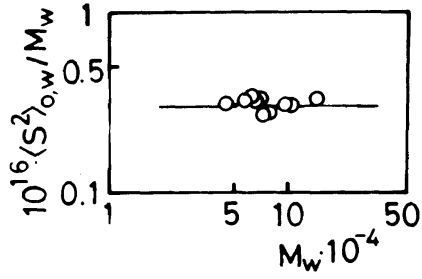

Figure 10. Unperturbed radius of gyration $\left\langle S^{2}\right\rangle_{0, w} /$ $M_{w}$ of cellulose acetate $(D S=0.49)$ in DMAc at $25^{\circ} \mathrm{C}$ : full line, of $\left\langle S^{2}\right\rangle_{0, w} / M_{w}=0.30 \times 10^{-16} \mathrm{~cm}^{2} \mathrm{~g}^{-1} \mathrm{~mol}$.

applied to the data of aqueous and formamide solutions in exactly the same manner, and $A$ was found to be $2.42 \times 10^{-8} \mathrm{~cm}$ and $2.97 \times 10^{-8} \mathrm{~cm}$ in water and formamide, respectively, as indicated in the second column of Table VI.

In general, the radius of gyration $\left\langle S^{2}\right\rangle^{1 / 2}$ can be expressed in terms of molecular weight by the relation

$$
\left\langle S^{2}\right\rangle^{1 / 2}=K_{\lambda} M^{(\lambda+1) / 2}
$$

where,

$$
\begin{gathered}
\lambda=a_{2}+2 \varepsilon \\
\varepsilon=\mathrm{d} \ln \alpha_{\mathrm{s}} / \mathrm{d} \ln M \\
a_{2}=\mathrm{d} \ln \left\langle S^{2}\right\rangle_{0} / M / \mathrm{d} \ln M
\end{gathered}
$$

For the CA $(D S=0.49)-D M A c$ system $\lambda$ was estimated to be 0.04 from eq g. Putting $\lambda=0.04$ and $a_{2}=0$ into eq 11 , we obtain $\varepsilon=0.02$, which is too small to detect experimentally from the molecular weight dependence of the expansion coefficient $\alpha_{\mathrm{s}}$. This means that the contribution of the excluded volume effect to the HMS exponent $a$ is negligibly small, being consistent with $\alpha_{\mathrm{s}} \lesssim 1.1-1.2$.

The exponent $a$ in eq 1 is given by the relation

$$
a=0.5+a_{\Phi}+3 \varepsilon+1.5 a_{2}
$$

Putting $a=0.60, a_{\Phi}=0.103$ and $\varepsilon=0.02$ for the CA $(D S=0.49)$-DMAc system into eq 14 , we obtain $a_{2}=-0.04$, which is too small to detect by experiment. The main factor contribution to the $a$ value is the partially free draining effect; the volume effect is apparently minor.

Figure 11 shows the plots of Baumann, Stockmayer-Fixman, and Kamide et al. for CA $(D S=0.49)$ in various solvents. The $A$ and $B$ values for CA $(D S=0.49)$ solutions in DMAc, obtained from the intercepts and slopes of the above plots,

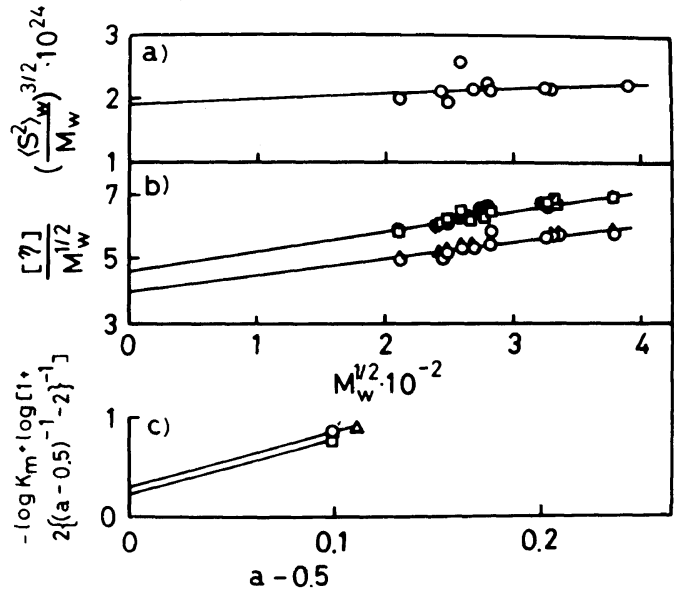

Figure 11. Baumann plot (a), Stockmayer-Fixman plot (b) and Kamide et al. plot (c) cellulose acetate $(D S=0.49)$ in DMAc $(\bigcirc)$, DMSO $(\triangle)$, water $(\square)$, and FA (O) at $25^{\circ} \mathrm{C}$.

Table V. Unperturbed chain dimension $A$, long-range

\begin{tabular}{|c|c|c|c|c|}
\hline \multirow{2}{*}{ Method } & $A \times 10^{8}$ & $B \times 10^{27}$ & \multirow{2}{*}{$\sigma$} & \multirow{2}{*}{$C_{\infty}$} \\
\hline & $\mathrm{cm}$ & $\mathrm{cm}^{3}$ & & \\
\hline $2 \mathrm{~B}\left(\alpha_{\mathrm{s}}\right.$ from $\left.\Psi\right)$ & 1.28 & - & 2.23 & 10.0 \\
\hline $2 \mathrm{C}\left(a_{2}=0\right)$ & 1.38 & 0.79 & 2.35 & 11.4 \\
\hline $2 \mathrm{E}\left(a_{2}=a_{\Phi}=0\right)$ & 1.12 & 3.70 & 1.91 & 7.5 \\
\hline $2 \mathrm{~F}\left(a_{2}=a_{\Phi}=0\right)$ & 1.14 & - & 1.94 & 7.8 \\
\hline Most probable & 1.33 & - & 2.29 & 10.7 \\
\hline
\end{tabular}
interaction parameter $B$, conformation parameter $\sigma$, and characteristic ratio $C_{\infty}$ of $\mathrm{CA}(D S=0.49)$ in DMAc at $25^{\circ} \mathrm{C}$

Table VI. Unperturbed-chain dimension $A$ of cellulose acetate $(D S=0.49)$ evaluated by method

\begin{tabular}{|c|c|c|c|c|c|}
\hline \multirow{2}{*}{ Solvents } & \multicolumn{3}{|c|}{$A \times 10^{8} / \mathrm{cm}$} & \multirow{2}{*}{$\frac{\sigma}{2 \mathrm{~B}}$} & \multirow{2}{*}{$\frac{C_{\infty}}{2 \mathrm{~B}}$} \\
\hline & $2 \mathrm{~B}$ & $2 \mathrm{E}$ & $2 \mathrm{~F}$ & & \\
\hline DMAc & 1.28 & 1.12 & 1.14 & 2.23 & 10.0 \\
\hline DMSO & - & 1.12 & 1.14 & - & - \\
\hline Water & 2.42 & 1.17 & 1.22 & 4.11 & 34.7 \\
\hline Formamide & 2.97 & 1.19 & 1.22 & 5.06 & 52.5 \\
\hline
\end{tabular}
$2 \mathrm{~B}, 2 \mathrm{E}$, and $2 \mathrm{~F}$ and conformation parameter $\sigma$ and characteristic ratio $C_{\infty}$, both estimated by method $2 \mathrm{~B}$ 
are summarized in Table V. In Table VI, the $A$ values in various other solvents, are tabulated. The prerequisites for the methods $2 \mathrm{E}$ and $2 \mathrm{~F}$ are $a_{2}=a_{\Phi}=0$. The latter is evidently not acceptable from an experimental point of view (see Figure 8). The methods $2 \mathrm{~B}$ and $2 \mathrm{C}$ are then preferred to methods $2 \mathrm{E}$ and $2 \mathrm{~F}$. The $A$ values calculated from methods $2 \mathrm{E}$ and $2 \mathrm{~F}$ are from some $20 \%$ (in DMAc) to $150 \%$ (in formamide) smaller than the most probable value, which is an average of the values obtained by the methods $2 \mathrm{~B}$ and $2 \mathrm{C}$. The large discrepancy in the $A$ values derived by the thermodynamic (methods $2 \mathrm{~B}$ and $2 \mathrm{C}$ ) and hydrodynamic (methods $2 \mathrm{E}$ and $2 \mathrm{~F}$ ) approaches can be accounted for by taking the draining effect into consideration.

The conformation parameter $\sigma$ and the characteristic ratio $C_{\infty}$ were calculated from the $A$ values in the conventional manner, and are given in Tables $\mathrm{V}$ and VI. $A=1.33 \times 10^{-8} \mathrm{~cm}, \sigma=2.29$, and $C_{\infty}=10.7$ were obtained as the most probable values for $\mathrm{CA}$ $(D S=0.49)$ in DMAc. The $\sigma$ value obtained here for CA $(D S=0.49)$ in DMAc is the minimum of these values in 25 systems of cellulose, amylose and their derivatives in solvents, ${ }^{5,6,15}$ and is not so different from those of the vinyl-type polymers.

The unperturbed-chain dimension $A$ depends on the dielectric constant of the solvent as is evident for CA in Figure 12. This dependence is less remarkable for CA $(D S=2.92)$, probably due to its less polar character. It is interesting that the $A$ value, extrapolated by $\varepsilon=1$, is larger for larger $D S$.

The effect of the degree of substitution on $A$ and $\sigma$ for CA in DMAc is demonstrated in Figure 13. The cellulose chain becomes the most rigid at a certain specific degree of substitution $(D S=2-2.5)$. In other words, CA $(D S=0.49)$ is more flexible, as opposed to our preliminary prediction, than CA $(D S=2.46)$ at least in DMAc.

\section{REFERENCES}

1. G. P. Pearson and W. R. Moore, Polymer, 1, 144 (1960).

2. G. V. Schulz and E. Penzel, Makromol. Chem., 112, 260 (1968).

3. E. Penzel and G. V. Schulz, Makromol. Chem., 113, 64 (1968).

4. E. Penzil and G. V. Schulz, Makromol. Chem., 162, 269 (1972).

5. K. Kamide, T. Terakawa, and Y. Miyazaki, Polym. J., 11, 285 (1979).

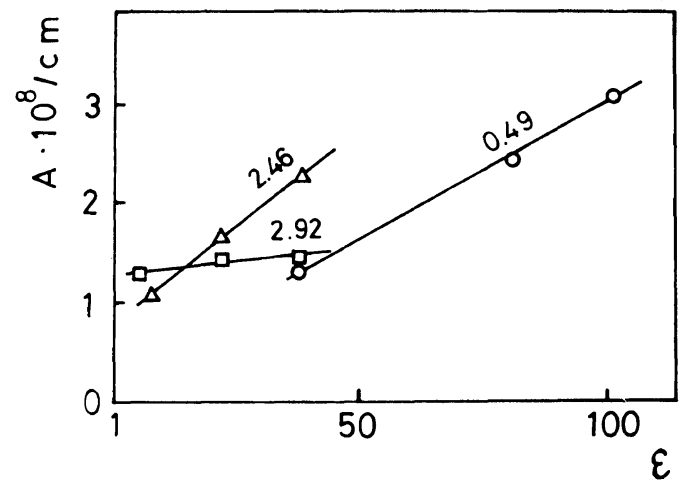

Figure 12. Dependence of the unperturbed chain dimensions $A$ of cellulose acetate on the dielectric constant $\varepsilon$ of the solvents: $\bigcirc, \mathrm{CA}(D S=0.49)$ (this work); $\triangle, \mathrm{CA}(D S=2.46)^{5} ; \square, \mathrm{CA}(D S=2.92) .^{6}$

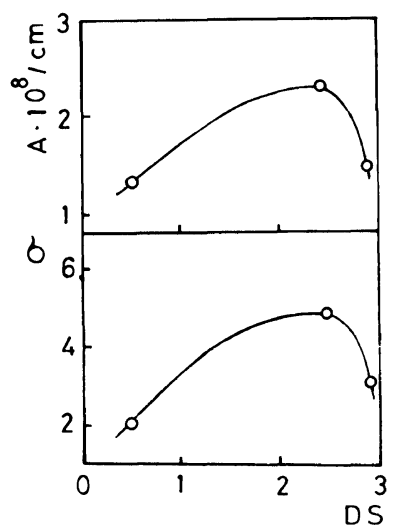

Figure 13. Effect of degree of substitution $(D S)$ on the unperturbed chain dimension A, conformation parameter $\sigma$ of cellulose acetate in DMAc at $25^{\circ} \mathrm{C}$.

6. K. Kamide, Y. Miyazaki, and T. Abe, Polym. J., 11, 523 (1979).

7. S. Ishida, H. Komatsu, T. Terakawa, Y. Miyazaki, and K. Kamide, Mem. Fac. Eng. Kanazawa Univ., 12, 103 (1979).

8. K. Kamide, Y. Miyazaki, and T. Abe, Makromol. Chem., 180, 2801 (1979).

9. K. Kamide and K. Okujima, Polym. J., 13, 127 (1981).

10. B. H. Zimm, J. Chem. Phys., 16, 1093 (1948).

11. B. H. Zimm, J. Chem. Phys., 16, 1099 (1948).

12. C. H. Malm, C. R. Tordyce, and H. A. Tanner, Ind. Eng. Chem., 34, 430 (1942).

13. J. A. Dean, Ed., "Lange's Handbook of Chemistry," twelfth ed, 10, McGraw-Hill, New York, N.Y., 1979, p 103.

14. K. Kamide, K. Okajima, and M. Saito, Polym. J., 13, 115 (1981). 


\section{Dilute Solution Properties of Water-Soluble CA}

15. K. Kamide and Y. Miyazaki, Polym. J., 10, 409 (1978).

16. M. Kurata and H. Yamakawa, J. Chem. Phys., 29, 311 (1958).

17. H. Baumann, J. Polym. Sci., C, No. 3, 1069 (1965).

18. W. H. Stockmayer and M. Fixman, J. Polym. Sci., C, No. 1, 137 (1963).

19. T. Kawai and K. Kamide, J. Polym. Sci., No. 54, 343 (1961).

20. K. Kamide and T. Kawai, Kobunshi Kagaku, 19, 441
(1962).

21. K. Kamide and T. Kawai, Kobunshi Kagaku, 20, 512 (1963)

22. K. Kamide and W. R. Moore, Kobunshi Kagaku, 21, 682 (1964).

23. K. Kamide and W. R. Moore, J. Polym. Sci., B, 2, 1029 (1964).

24. K. Kamide, Y. Inamoto, and G. Livingstone, Kobunshi Kagaku, 23, 1 (1966). 